\title{
Ropivacaine plasma levels following local infiltration analgesia for primary total hip arthroplasty
}

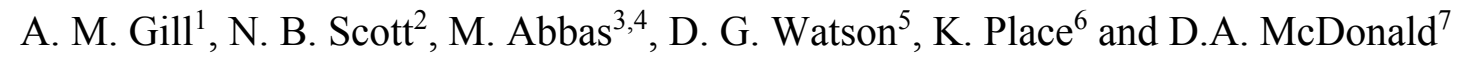

1 Consultant, Department of Anaesthesia, 7 Clinical Researcher, Department of

Rehabilitation, Golden Jubilee National Hospital, Clydebank

2 Consultant, Department of Anaesthesia, Hamad Medical Corporation, Doha, State of Qatar

3 PhD Student, Department of Pharmacy, Abdul Wali Khan University, Mardan, Pakistan.

$4 \mathrm{PhD}$ Student, 5 Reader, Institute of Pharmacy and Biomedical Sciences, University of

Strathclyde, Glasgow

6 Consultant, Department of Anaesthesia, Royal Berkshire Foundation Trust, Reading

Correspondence to: A. M. Gill

Email: Mike.Gill@gjnh.scot.nhs.uk

Short title: Ropivacaine plasma levels following local infiltration analgesia

Summary 
We measured total and free plasma concentrations of ropivacaine following high-volume, high-dose local infiltration analgesia in nineteen patients aged 65 years or over undergoing unilateral total hip arthroplasty. The patients received $180 \mathrm{ml}(360 \mathrm{mg})$ of ropivacaine which was injected into the deep and peri-capsular tissues, the gluteal muscles and fascia lata, and the subcutaneous tissues and skin. Patients were monitored for clinical symptoms and signs of systemic local anaesthetic toxicity. Total levels of plasma ropivacaine varied from $0.081-$ $1.707 \mu \mathrm{g} \cdot \mathrm{ml}^{-1}$ (mean $0.953 \mu \mathrm{g} \cdot \mathrm{ml}^{-1}$ ). Free levels of plasma ropivacaine varied from $0.000-$ $0.053 \mu \mathrm{g} \cdot \mathrm{ml}^{-1}$ (mean $0.024 \mu \mathrm{g} \cdot \mathrm{ml}^{-1}$ ). No samples reached the toxic threshold for venous ropivacaine concentration, although four patients exhibited mild symptoms consistent with local anaesthetic toxicity. One patient had episodes of complete heart block on ECG monitoring but plasma ropivacaine levels were below toxic levels. We conclude that plasma levels for ropivacaine associated with toxicity in a volunteer population (total $2.2 \mu \mathrm{g} \cdot \mathrm{ml}^{-1}$, free $0.15 \mu \mathrm{g} \cdot \mathrm{ml}^{-1}$ ) are not reached during local infiltration analgesia for hip arthroplasty in elderly patients. 
Early mobilisation following total hip arthroplasty can facilitate patient recovery and minimise complications following surgery $[1,2]$. Both central neuraxial blockade and peripheral nerve block can cause motor deficit and this has significant disadvantages for patients in terms of mobilisation. Therefore, alternative analgesic techniques have been developed to support both effective pain relief and patient recovery [3 - 5].

Local infiltration analgesia is an increasingly popular technique for the management of early postoperative pain although concerns have been expressed regarding the safety of injecting large volumes of local anaesthetic into hip or knee joints during orthopaedic procedures [6].

The aim of this descriptive observational study was to analyse the total and free plasma concentrations of ropivacaine following high-volume, high-dose local infiltration analgesia in patients undergoing total hip arthroplasty. In addition to plasma levels of ropivacaine, we also monitored patients for symptoms or signs of local anaesthetic toxicity.

\section{Methods}

The study protocol was approved by the regional ethics committee (November 2011; 11/WS/0086) and was conducted in accordance with the Declaration of Helsinki. Twenty patients gave written and informed consent for inclusion in the study. We included patients $\geq 65$ y having unilateral total hip arthroplasty. The exclusion criteria were sensitivity or allergy to amide anaesthetics, patients who were unable to give consent and those with cardiac, respiratory, hepatic or renal failure. If the planned operation or anaesthetic technique changed after consent had been obtained the patient was withdrawn from the study.

All patients followed the standardised enhanced recovery programme of our institution. This comprises a package of care including pre-operative education, multimodal 
analgesia and accelerated rehabilitation. The anaesthetic/analgesic technique consists of regional anaesthesia, pre- and post-operative analgesia and the use of local infiltration of ropivacaine during hip arthroplasty.

All patients were admitted the day before surgery and received oral temazepam $20 \mathrm{mg}$ and gabapentin $600 \mathrm{mg}$ at night. On the day of surgery, patients received oral temazepam 20 $\mathrm{mg}$, paracetamol $1 \mathrm{~g}$ and dexamethasone $10 \mathrm{mg} 2 \mathrm{~h}$ pre-operatively. A $16 \mathrm{G}$ intravenous cannula (Becton Dickinson, Swindon, UK) was sited pre-operatively and a baseline venous blood sample was taken. Heart rate and non-invasive blood pressure monitoring were instituted in the pre-operative reception area in addition to 24-h ECG monitoring (Seer Light Recorder, GE, Amersham, UK) to enable rhythm analysis.

Spinal anaesthesia was performed at L3-4 using $2.5 \mathrm{ml}$ hyperbaric bupivacaine 5 mg.ml ${ }^{-1}$. All patients received propofol sedation using a target-controlled infusion pump and were monitored by pulse oximetry in addition to those already commenced.

At the beginning of the surgical procedure, tranexamic acid $2.5 \mathrm{~g}$ was administered. Local anaesthetic infiltration was performed by the surgeon intraoperatively using $180 \mathrm{ml}$ of plain ropivacaine $2 \mathrm{mg} \cdot \mathrm{ml}^{-1}$. Circumferential infiltration of the deep and peri-capsular tissues with up to $80 \mathrm{ml}$ was followed by infiltration of the gluteal muscles and fascia lata with approximately $70 \mathrm{ml}$. Finally, $30 \mathrm{ml}$ was used to infiltrate the subcutaneous tissues and skin to complete the total of $180 \mathrm{ml}$. Wound drains were not used.

Venous blood samples were taken at 5, 10, 15, 20, 25, and $30 \mathrm{~min}$, then 1,4 and $24 \mathrm{~h}$ following injection of local anaesthetic. Patients were asked about the presence of symptoms of local anaesthetic toxicity (light-headedness, blurred vision, tingling of the tongue or visual/auditory disturbances). In addition, more significant signs such as loss of consciousness, seizures or cardiac arrest were recorded. Intermittent heart rate and blood pressure readings and continuous 24-h ECG monitoring with rhythm analysis were recorded 
during the period of venous blood sampling. Completion of the patient's involvement in the study occurred following the final blood sample at $24 \mathrm{~h}$ and removal of the ECG monitor.

Blood samples were centrifuged immediately after sampling by the Biochemical laboratory at the study site before being transferred to the Institute of Pharmacy and Biomedical Sciences, University of Strathclyde, Glasgow. Samples were analysed by the buffering of blood samples before separation of free and bound ropivacaine using equilibrium dialysis [7]. When analysis could not proceed immediately, samples were frozen, stored at minus $20^{\circ} \mathrm{C}$ and then subsequently thawed prior to testing. The measurement of standards and samples was performed using liquid chromatography-mass spectrometry on a Surveyor HPLC system combined with an Orbitrap mass spectrometer (Thermo Fisher Scientific, Hemel Hempstead, UK). The results were analysed with descriptive statistics using GraphPad Prism Mac V6.0b software (GraphPad Software, San Diego, California). The maximum tolerated venous concentrations of ropivacaine were set at $2.2 \mu \mathrm{g} \cdot \mathrm{ml}^{-1}$ for total plasma ropivacaine concentration and $0.15 \mu \mathrm{g} \cdot \mathrm{ml}^{-1}$ for free plasma ropivacaine concentration as previously published in a volunteer population [8].

\section{Results}

The data collection took place from August 2012 to March 2013. Twenty patients gave consent for inclusion in the study. Following consent, one patient (H4) was found not to meet the inclusion criteria and was excluded. The remaining 19 patients all completed the study. The mean (SD) patient age was $76.0(6.3) \mathrm{y}$, weight $72.5(15.3) \mathrm{kg}$ and body mass index $27.3(4.2) \mathrm{kg} \cdot \mathrm{m}^{-2}$. Two patients were ASA grade 1,13 were grade 2 and four were grade 3. Patient characteristics and peak concentration (Cmax) data are presented in Table 1. A total of 184 blood samples were collected for analysis. Six samples were missed due to venesection difficulties. 
Excluding baseline samples, total plasma ropivacaine levels varied from 0.081 $1.707 \mu \mathrm{g} \cdot \mathrm{ml}^{-1}$ with a mean of $0.953 \mu \mathrm{g} \cdot \mathrm{ml}^{-1}$ (Fig. 1a). The free levels of ropivacaine varied from $0.000-0.053 \mu \mathrm{g} \cdot \mathrm{ml}^{-1}$ with a mean of $0.024 \mu \mathrm{g} \cdot \mathrm{ml}^{-1}$ (Fig. 1b). The maximum doses were below the toxic thresholds of $2.2 \mu \mathrm{g} \cdot \mathrm{ml}^{-1}$ for total and $0.15 \mu \mathrm{g} \cdot \mathrm{ml}^{-1}$ for free plasma ropivacaine concentration established in a volunteer population [8].

Four patients exhibited mild symptoms consistent with local anaesthetic toxicity. These were transient dizziness, nausea and blurred vision and occurred at the times specified in Table 2. All symptoms resolved without treatment.

One patient was found on ECG monitoring to have had episodes of complete heart block during the study period. Analysis showed that maximum plasma ropivacaine levels during these episodes were $1.12 \mu \mathrm{g} \cdot \mathrm{ml}^{-1}$ (total) and $0.03 \mu \mathrm{g} \cdot \mathrm{ml}^{-1}$ (free). Subsequent telemetry over the postoperative stay revealed further episodes of complete heart block and the patient underwent implantation of a permanent pacemaker.

\section{Discussion}

This study aimed to assess the plasma ropivacaine concentrations during local infiltration analgesia for hip arthroplasty. Our results demonstrated that neither the total nor free levels of plasma ropivacaine reached potentially toxic venous concentrations of ropivacaine.

Following the description of the local infiltration analgesia technique for hip and knee arthroplasty [3], several papers were published which reported equivalent or improved outcomes when compared to other regional analgesic techniques $[9,10]$. Conversely, there remains debate regarding whether local infiltration analgesia for hip arthroplasty provides additional pain relief within a multimodal analgesic regimen [11]. The effectiveness of local 
infiltration analgesia in terms of pain scoring and postoperative functional recovery lies outside the scope of this study.

Studies published for local infiltration analgesia have primarily concentrated on plasma ropivacaine concentrations after knee arthroplasty [12-14]. In addition, although there are studies on hip surgery patients that show ropivacaine levels below the accepted toxic limits [15], it has been recognised as a limitation that free (or unbound) levels were not analysed. To our knowledge only one previous study has reported the plasma concentrations following hip arthroplasty with the use of levobupivacaine, but data for free ropivacaine concentration levels are not available [16]. The free proportion of a drug is more likely to be predictive of toxicity than the total concentration, therefore we feel that this study provides evidence that local infiltration with ropivacaine can be used safely in elderly patients undergoing total hip arthroplasty.

Study patients received a total volume of $180 \mathrm{ml} 0.2 \%$ ropivacaine. The mean dose received was $5.2 \mathrm{mg} . \mathrm{kg}^{-1}$ with a range from 3.5-6.8 mg. $\mathrm{kg}^{-1}$. All patients received a dose greater than the manufacturers' recommended maximum dose of $3 \mathrm{mg} \cdot \mathrm{kg}^{-1}$. Recent studies have demonstrated symptomatic local anaesthetic toxicity following an injection of ropivacaine into the layers of the abdominal wall [17]. Our results give support to the hypothesis that local anaesthetic plasma levels may vary depending on the tissues into which the drug is administered [18] and that the periarticular hip region is a relatively safe anatomical compartment for large volumes of local anaesthetic. However, the optimal dose for local infiltration analgesia in hip arthroplasty is as yet unknown and should be subject to further studies. We used ropivacaine without any additives although there is discussion on the relative merits of administering ropivacaine with adjuvants such as adrenaline or ketorolac [19]. Plasma ropivacaine levels are unlikely to be higher with adrenaline-containing 
solutions, although it should be noted that ropivacaine has intrinsic vasoconstrictor properties.

The study was designed to detect potential signs of local anaesthetic toxicity. We found that five patients had symptoms or signs consistent with toxic plasma levels of ropivacaine. In these patients, the symptoms observed could have been attributable to factors other than ropivacaine toxicity such as the effects of sedation or the use of opioid analgesia. The free level of ropivacaine at the time of the onset of symptoms remained below the mean venous toxic level. The patient who showed complete heart block on the ECG monitor continued to display conduction defects on telemetry in the days following surgery. Subsequent investigation revealed that the patient had experienced dizziness in the preceding months often associated with emesis. Before discharge after surgery, the patient underwent implantation of a permanent pacemaker for complete heart block presumed secondary to cardiac sarcoidosis.

This study has a number of limitations and implications. Firstly, the toxic reference levels used were provided from a study performed on healthy young volunteers. It is accepted that the toxic thresholds may be different in elderly patients although, to our knowledge, such toxicity data does not exist. Elderly patients may have reduced organ blood flow and function that can decrease the clearance of local anaesthetic drugs [20]. However, this is more relevant when considering repeated doses of local anaesthetic. In addition, although peak plasma concentrations and plasma protein binding are similar in elderly people and young adults [21], axonal function deteriorates and nerve morphology changes with advancing age [22]. This may result in toxic symptoms or signs at lower plasma levels than would be the case with young adults.

It is possible that the injection technique might not deliver ropivacaine to the intended anatomical sites during the procedure. Injection into more vascular structures could 
potentially have altered the pharmacokinetic profiles seen, leading to more rapid uptake into the plasma; however we did not observe this.

Target-controlled propofol sedation was used for all study patients. Subtle symptoms or signs of local anaesthetic toxicity may have been masked in the immediate postoperative period due to sedation. Equally, the use of sedation may have been responsible for the transient dizziness, blurred vision and nausea observed.

The use of ECG Holter monitoring provided heart rate analysis throughout the study period and allowed the detection of dysrhythmias that may have been caused by toxic local anaesthetic plasma levels. However, it may not have been sufficiently sensitive to detect the more subtle changes in ECG morphology that can be associated with local anaesthetic toxicity such as P-R prolongation or QRS widening. Other studies have used serial ECG measurements to determine whether cardiac electrophysiology is altered by local anaesthetics $[8]$.

In conclusion, this study shows that plasma levels of ropivacaine associated with toxicity in a volunteer population are not reached during high-volume, high-dose local infiltration analgesia using $360 \mathrm{mg}$ of ropivacaine for total hip arthroplasty in elderly patients.

\section{Acknowledgements}

We are grateful to Shiona McKelvie at the Golden Jubilee National Hospital for her assistance as a participating investigator and for providing care for study patients.

\section{Declaration of interest}


No conflicts of interest were declared by the authors. This work was supported by B.Braun, Sheffield, UK who provided financial assistance that enabled the purchase of equipment and consumable materials for blood sample testing. 


\section{References}

1. Kehlet H, Dahl JB. Anaesthesia, surgery, and challenges in postoperative recovery. Lancet 2003; 362: 1921-8.

2. Larsen K, Sørensen OG, Hansen TB, Thomsen PB, Søballe K. Accelerated perioperative care and rehabilitation intervention for hip and knee replacement is effective: a randomized clinical trial involving 87 patients with 3 months of follow-up. Acta Orthopaedica 2008; 79: 149-59.

3. Kerr DR, Kohan L. Local infiltration analgesia: a technique for the control of acute postoperative pain following knee and hip surgery: a case study of 325 patients. Acta Orthopaedica 2008; 79: 174-83.

4. Busch CA, Whitehouse MR, Shore BJ, MacDonald SJ, McCalden RW, Bourne RB. The efficacy of periarticular multimodal drug infiltration in total hip arthroplasty. Clinical Orthopaedics and Related Research 2010; 468: 2152-9.

5. Andersen LJ, Poulsen T, Krogh B, Nielsen T. Postoperative analgesia in total hip arthroplasty: a randomized double-blinded, placebo-controlled study on peroperative and postoperative ropivacaine, ketorolac and adrenaline wound infiltration. Acta Orthopaedica 2007; 78: 187-92.

6. McCartney CJL, McLeod GA. Local infiltration analgesia for total knee arthroplasty. British Journal of Anaesthesia 2011; 107: 487-9.

7. Abbas M, Ahmad L, Shah Y, Gill M, Watson DG. Development of a method to measure free and bound ropivacaine in human plasma using equilibrium dialysis and hydrophilic interaction chromatography couple to high resolution mass spectrometry. Talanta $2013 ; \mathbf{1 1 7}$ : $60-3$. 
8. Knudsen K, Beckman Suurkula M, Blomberg S, Sjovall J, Edvardsson N. Central nervous and cardiovascular effects of i.v. infusions of ropivacaine, bupivacaine and placebo in volunteers. British Journal of Anaesthesia 1997; 78: 507-14.

9. Andersen KV, Bak M, Christensen BV, Harazuk J, Pedersen NA, Søballe K. A randomized, controlled trial comparing local infiltration analgesia with epidural infusion for total knee arthroplasty. Acta Orthopaedica 2010; 81: 606-10.

10. Spreng UJ, Dahl V, Hjall A, Fagerland MW, Ræder J. High-volume local infiltration analgesia combined with intravenous or local ketorolac+morphine compared with epidural analgesia after total knee arthroplasty. British Journal of Anaesthesia 2010; 105: 675-82. 11. Lunn TH, Husted H, Solgaard S, et al. Intraoperative local infiltration analgesia for early analgesia after total hip arthroplasty: a randomized, double-blind, placebo-controlled trial. Regional Anesthesia and Pain Medicine 2011; 36: 424-9.

12. Vendittoli P-A, Makinen P, Drolet P, et al. A multimodal analgesia protocol for total knee arthroplasty: a randomized, controlled study. The Journal of Bone and Joint Surgery 2006; 88: $282-9$.

13. Essving P, Axelsson K, Kjellberg J, Wallgren Ö, Gupta A, Lundin A. Reduced morphine consumption and pain intensity with local infiltration analgesia (LIA) following total knee arthroplasty. A randomized double-blind study involving 48 patients. Acta Orthopaedica 2010; 81: 354-60.

14. Busch CA, Shore BJ, Bhandari R, et al. Efficacy of periarticular multimodal drug injection in total knee arthroplasty: a randomized trial. The Journal of Bone and Joint Surgery 2006; 88: 959-63.

15. Bianconi M, Ferraro L, Traina GC, et al. Pharmacokinetics and efficacy of ropivacaine continuous wound instillation after joint replacement surgery. British Journal of Anaesthesia 2003; 91: 830-5. 
16. Rikalainen-Salmi R, Förster JG, Mäkelä K, et al. Local infiltration analgesia with levobupivacaine compared with intrathecal morphine in total hip arthroplasty patients. Acta Anaesthesiologica Scandinavica 2012; 56: 695-705.

17. Griffiths JD, Le NV, Grant S, Bjorksten A, Hebbard P, Royse C. Symptomatic local anaesthetic toxicity and plasma ropivacaine concentrations after transversus abdominis plane block for Caesarean section. British Journal of Anaesthesia 2013; 110: 996-1000.

18. Heavner JE. Let's abandon blanket maximum recommended doses of local anesthetics. Regional Anesthesia and Pain Medicine 2004; 29: 524.

19. Raeder JC. Local infiltration analgesia for pain after total knee replacement surgery: a winner or just a strong runner-up? Anesthesia and Analgesia 2011; 113: 684-6.

20. Bowdle AT, Freund PR, Slattery JT. Age-dependent lidocaine pharmacokinetics during lumbar peridural anesthesia with lidocaine hydrocarbonate or lidocaine hydrochloride. Regional Anesthesia and Pain Medicine 1986; 11: 123-7.

21. Veering BT, Burm AGL, Gladines MP, Spierdijk J. Age does not influence the serum protein binding of bupivacaine. British Journal of Clinical Pharmacology 1991; 32: 501-3. 22. Kurokawa Y, Mimori Y, Tanaka E, Kohriyama T, Nakamura S. Age-related change in peripheral nerve conduction: compound muscle action potential duration and dispersion. Gerontology 1999; 45: 168-73. 
Table 1. Patient characteristics and peak plasma ropivacaine concentration (Cmax) data in 19 cases of local infiltration analgesia.

$\begin{array}{llllllll}\text { Patient } & \begin{array}{l}\text { Age } \\ (\mathrm{y})\end{array} & \begin{array}{l}\text { Height } \\ (\mathrm{m})\end{array} & \begin{array}{l}\text { Weight } \\ (\mathrm{kg})\end{array} & \begin{array}{l}\text { BMI } \\ \left(\mathrm{kg} \cdot \mathrm{m}^{-2}\right)\end{array} & \begin{array}{l}\text { Ropivacaine } \\ \text { dose } \\ \left(\mathrm{mg} \cdot \mathrm{kg}^{-1}\right)\end{array} & \begin{array}{l}\text { Total } \\ \left(\mu \mathrm{g} \cdot \mathrm{ml}^{-1}\right)\end{array} & \begin{array}{l}\text { Free } \\ \left(\mu \mathrm{g} \cdot \mathrm{ml}^{-1}\right)\end{array} \\ \mathrm{H} 1 & 72.9 & 1.61 & 86 & 33.2 & 4.2 & 1.707 & 0.028 \\ \text { H2 } & 81.0 & 1.64 & 66 & 24.5 & 5.5 & 1.593 & 0.053 \\ \text { H3 } & 75.5 & 1.61 & 75 & 28.9 & 4.8 & 1.342 & 0.040 \\ \text { H5 } & 65.3 & 1.59 & 81 & 32.0 & 4.4 & 1.642 & 0.039 \\ \text { H6 } & 67.8 & 1.56 & 69 & 28.4 & 5.2 & 0.722 & 0.014 \\ \text { H7 } & 80.7 & 1.54 & 64 & 27.0 & 5.6 & 1.149 & 0.030 \\ \text { H8 } & 65.6 & 1.98 & 101 & 25.8 & 3.6 & 0.790 & 0.026 \\ \text { H9 } & 75.9 & 1.50 & 68 & 30.2 & 5.3 & 1.394 & 0.022 \\ \text { H10 } & 78.5 & 1.68 & 103 & 36.5 & 3.5 & 0.797 & 0.037 \\ \text { H11 } & 80.0 & 1.70 & 66 & 22.8 & 5.5 & 0.944 & 0.034 \\ \text { H12 } & 80.1 & 1.77 & 91 & 29.0 & 4.0 & 1.004 & 0.014 \\ \text { H13 } & 82.3 & 1.73 & 89 & 29.7 & 4.0 & 0.958 & 0.041 \\ \text { H14 } & 72.8 & 1.57 & 61 & 24.7 & 5.9 & 1.370 & 0.041 \\ \text { H15 } & 68.8 & 1.64 & 72 & 26.8 & 5.0 & 0.911 & 0.024 \\ \text { H16 } & 72.8 & 1.50 & 56 & 24.9 & 6.4 & 0.996 & 0.027 \\ \text { H17 } & 90.0 & 1.50 & 55 & 24.4 & 6.5 & 1.189 & 0.035 \\ \text { H18 } & 74.7 & 1.59 & 59 & 23.3 & 6.1 & 1.351 & 0.038 \\ \text { H19 } & 77.9 & 1.72 & 53 & 17.9 & 6.8 & 1.268 & 0.030 \\ \text { H20 } & 81.1 & 1.49 & 62 & 27.9 & 5.8 & 1.061 & 0.040\end{array}$


Table 2. Details of four patients exhibiting potential symptoms of systemic toxicity with concurrent total and free plasma ropivacaine concentrations.

\begin{tabular}{lllll}
\hline Patient & Symptom & Time reported & \multicolumn{2}{l}{ Plasma Levels $\left(\mu \mathrm{g} . \mathrm{ml}^{-1}\right)$} \\
\hline H5 & Dizziness & $25 \mathrm{~min}$ & 1.474 & 0.036 \\
H7 & Nausea & $30 \mathrm{~min}$ & 1.149 & 0.028 \\
H7 & Blurred vision & $4 \mathrm{~h}$ & 0.943 & 0.012 \\
H15 & Dizziness & $4 \mathrm{~h}$ & 0.851 & 0.019 \\
H6 & Blurred vision & $24 \mathrm{~h}$ & 0.722 & 0.008 \\
\hline
\end{tabular}


Caption to figure 1

Figure 1. Plasma ropivacaine concentrations following local infiltration analgesia (a) total (b) free. Values are mean (line), SD (error bars).
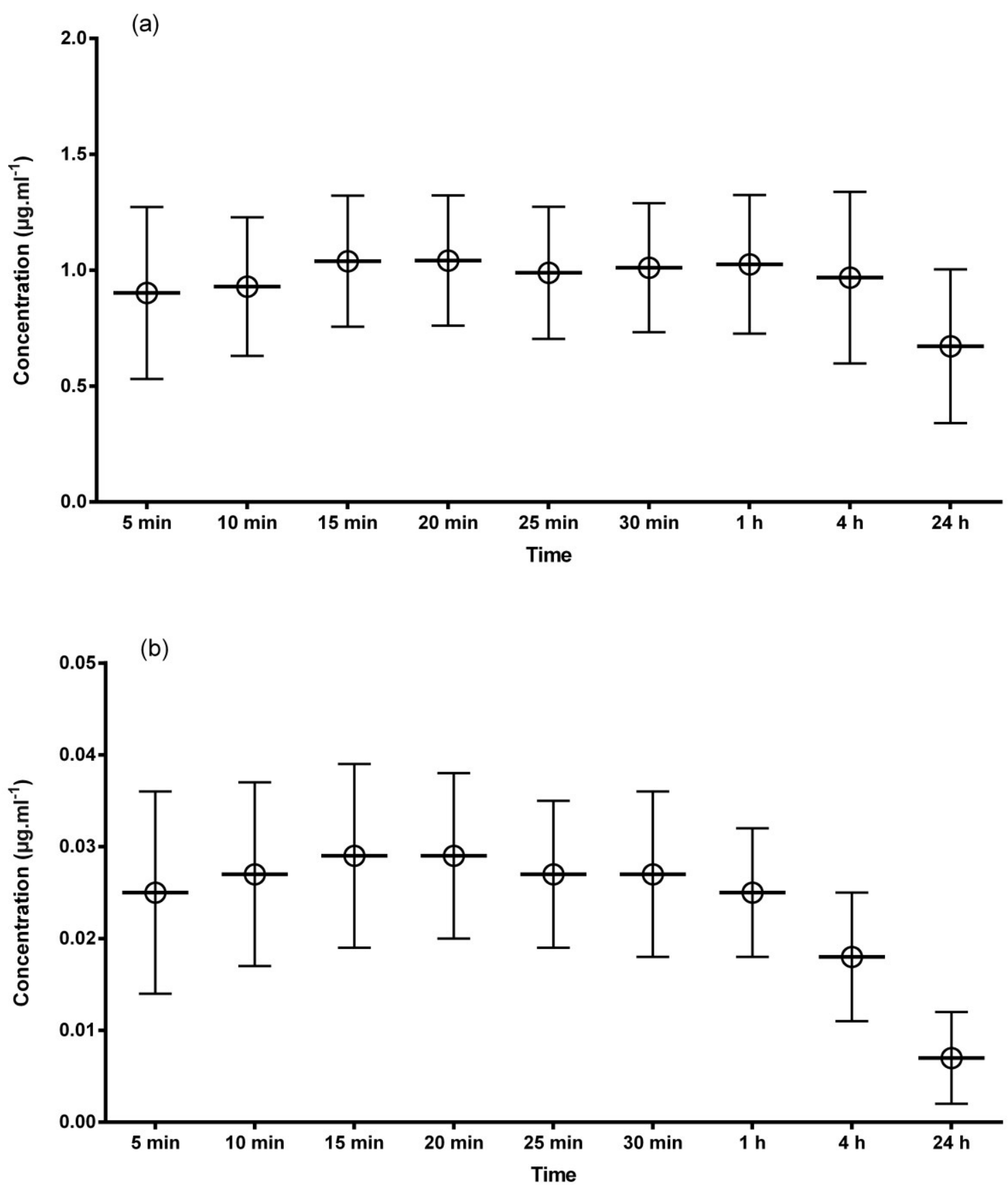
